\title{
FACTORES QUE INFLUYEN EN LA SATISFACCIÓN DE ESTUDIANTES UNIVERSITARIOS DE GRADO AL HACER USO DEL ENTORNO VIRTUAL DE APRENDIZAJE SAKAI. ANÁLISIS UTILIZANDO EL MODELO DE ACEPTACIÓN TECNOLÓGICA TAM EXTENDIDO
}

\author{
Antonio Paños Álvarez ${ }^{1}$ y Carolina López Nicolás ${ }^{2}$ \\ Departamento de Organización de Empresas y Finanzas, Universidad de Murcia, Campus de Espinardo, 30100 Murcia, \\ España \\ ${ }^{1}+34868887237$ \\ ${ }^{2}+34868883762$
}

\section{RESUMEN}

El estudio analiza la influencia del apoyo técnico, la utilidad percibida, la facilidad de uso y la interrelación con los contenidos digitales, como determinantes del desempeño percibido (rendimiento) de alumnos universitarios de Grado de la asignatura de Administración de Empresas al utilizar el entorno virtual de aprendizaje SAKAI como herramienta de innovación docente y de mejora del proceso de enseñanza-aprendizaje de dichos alumnos. En el estudio realizado sólo 3 factores afectan positiva y significativamente el rendimiento, siendo el más influyente la utilidad percibida. La interrelación con los contenidos digitales no resultó significativa.

\section{PALABRAS CLAVE}

Innovación Educativa, Entorno Virtual de Aprendizaje, Universidad, SAKAI

\section{INTRODUCCIÓN}

El uso de entornos virtuales de aprendizaje se ha extendido entre las universidades de todo el mundo (Ong et al., 2017), aunque la investigación sobre su uso a día de hoy es aún limitada y está en curso (Attardi y Rogers, 2015). Como se comenta en su página web, el proyecto SAKAI es un sistema LCMS (Learning Content Management System) que integra a más de 4 millones de usuarios educativos en actividades de enseñanza-aprendizaje e investigación. Las últimas versiones de SAKAI contienen múltiples herramientas y mecanismos de comunicación (Bainbridge et al.,2015) como por ejemplo: mensajes, recursos, tareas, foros, exámenes, evaluaciones, correcciones, videos, enlaces web, tests, lessons builder, etc. Esta diversidad de aplicaciones permite que las aulas virtuales basadas en SAKAI faciliten la implementación de metodologías docentes innovadoras como la clase invertida, le enseñanza basada en la evidencia y otras (Mclaughlin \& Rhoney, 2015; Ong et al., 2018).

Investigaciones anteriores se han centrado en analizar la aceptación de nuevas tecnologías aplicadas a la docencia en el entorno virtual Moodle (Arteaga y Duarte, 2010) o en cursos universitarios online (Kuo et al., 2014). La mayoría de estos estudios (por ejemplo, Cheng, 2011 o Arteaga y Duarte, 2010) coinciden en plantear modelos teóricos basados en el Technology Accepatance Model (TAM) de Davis et al. (1989) y sus sucesivas ampliaciones en trabajos como los de Arteaga y Duarte (2010) o Cheng (2011).

El proyecto SAKAI que se inició en las Universidades de Michigan e Indiana y se consolidó con la Fundación Mellon en 2004, está presente en más de 100 universidades en todo el mundo. En España la plataforma SAKAI está presente en universidades como la Complutense de Madrid, la Oberta de Catalunya y en la Universidad de Murcia, de aquí nuestro interés en analizar el entorno virtual SAKAI. Las características y recursos de las plataformas Moodle y SAKAI en sus versiones más recientes son similares (Arnaldos et al., 2015), pero, sin embargo los estudios sobre esta plataforma son más escasos que para el caso de otras como Moodle. 


\section{FUNDAMENTOS TEÓRICOS DEL ESTUDIO}

A partir de la literatura existente, nuestra investigación plantea y valida empíricamente un modelo basado en el TAM introducido por Davis et al. (1989). El modelo TAM, ampliamente consensuado y su versión posterior TAM2 (Venkatesh y Davis, 2000), explican la aceptación y uso de tecnologías de la información con base en la Teoría de la Acción Razonada (Fishbein y Ajzen, 1975) y establece la utilidad percibida y la facilidad de uso percibida como variables explicativas. Estudios más recientes y centrados en el ámbito de la educación universitaria, han ido extendiendo el modelo e incorporando otras variables explicativas, como el soporte técnico (Ngai et al., 2007; Arteaga y Duarte, 2010) o la interaccciones del estudiante con los contenidos digitales (Kuo et al., 2014). En este estudio se introduce otro paradigma, la satisfacción del usuario definido a partir del desempeño percibido (perceived performance) como variable dependiente (Mckinney et al., 2002), en lugar de la actitud hacia el uso de la tecnología. Nuestro modelo trata de explicar la utilización del aula virtual SAKAI entre los alumnos de una universidad española a partir de cuatro factores determinantes: apoyo técnico (technical support), interacciones del estudiante con los contenidos (learner-content interactions) utilidad percibida (perceived usefulness) y facilidad de uso (perceived ease of use).

\section{METODOLOGÍA}

Las hipótesis planteadas se contrastan empíricamente en una muestra de 114 estudiantes de la asignatura de Administración de Empresas de primer curso de los Grados en ADE y en Comunicación Audiovisual en la Universidad de Murcia. Las herramientas docentes tecnológicas utilizadas por ambos grupos en el Aula Virtual SAKAI son recursos (repositorio de documentos), mensajes, tareas, foros, enlaces web y calificaciones. El cuestionario es online y contiene escalas de medida de las variables del estudio extraídas de investigaciones anteriores: desempeño percibido de Cheng (2011), interacciones con los contenidos de Kuo et al. (2014) y las restantes (apoyo técnico, utilidad percibida y facilidad de uso) de Arteaga y Duarte (2010). El análisis factorial y el alfa de Cronbach indican que las medidas utilizadas son válidas y fiables, salvo para el caso de las interacciones del estudiante con los contenidos del aula virtual $(\alpha$ de Cronbach $<0,8)$. Por ello, se descartó la inclusión de este factor en el modelo. El análisis factorial de componentes principales y rotación Varimax (Tabla 1) confirmó 4 factores (varianza explicada=67,18\%), que coinciden con los cuatro constructos (desempeño percibido, apoyo técnico, utilidad percibida y facilidad de uso) y cada ítem presenta fuertes cargas solo en su factor. Los índices de alfa de Cronbach superan ampliamente el nivel de 0,8.

Tabla 1. Resultados del análisis factorial y fiabilidad

\begin{tabular}{|l|c|c|c|c|}
\hline \multicolumn{2}{|l|}{ Matriz de componente rotado } \\
\cline { 2 - 4 } & $\begin{array}{c}\text { Utilidad } \\
\text { percibida } \\
\alpha=0,87\end{array}$ & $\begin{array}{c}\text { Apoyo técnico } \\
\alpha=0,82\end{array}$ & $\begin{array}{c}\text { Facilidad de } \\
\text { uso } \alpha=0,84\end{array}$ & $\begin{array}{c}\text { Desempeño } \\
\text { percibido } \\
\alpha=0,82\end{array}$ \\
\hline $\begin{array}{l}\text { 1. El Aula Virtual proporciona asistencia cuando hay un } \\
\text { problema técnico }\end{array}$ & & 0,739 & & \\
\hline $\begin{array}{l}\text { 2. En el Aula Virtual existe una atención en línea } \\
\text { disponible en todo momento }\end{array}$ & & 0,707 & & \\
\hline $\begin{array}{l}\text { 3. En el Aula Virtual se pueden hacer consultas por correo } \\
\text { electrónico cuando existe un problema técnico }\end{array}$ & & 0,776 & & \\
\hline $\begin{array}{l}\text { 4. En el Aula Virtual se pueden hacer consultas a través de } \\
\text { la propia web cuando existe un problema técnico }\end{array}$ & & 0,792 & & \\
\hline $\begin{array}{l}\text { 5. En general, opino que el Aula Virtual ofrece un buen } \\
\text { soporte técnico }\end{array}$ & & 0,697 & & \\
\hline 6. El Aula Virtual me ayuda a aprender con más facilidad & 0,596 & & & \\
\hline 7. El Aula Virtual mejora mi rendimiento académico & 0,865 & & & \\
\hline $\begin{array}{l}\text { 8. El Aula Virtual hace que mi aprendizaje de la asignatura } \\
\text { sea más eficaz }\end{array}$ & 0,771 & & & \\
\hline $\begin{array}{l}\text { 9. El Aula Virtual hace que aprender en la Universidad sea } \\
\text { más fácil }\end{array}$ & 0,778 & & & \\
\hline
\end{tabular}




\begin{tabular}{|c|c|c|c|}
\hline $\begin{array}{l}\text { 10. El Aula Virtual me da más control sobre mi proceso de } \\
\text { aprendizaje de la asignatura }\end{array}$ & 0,659 & & \\
\hline $\begin{array}{l}\text { 11. En general, considero, que el Aula Virtual es ventajosa } \\
\text { para mi aprendizaje de la asignatura }\end{array}$ & 0,653 & & \\
\hline 12. Aprender a usar el Aula Virtual es fácil & & 0,867 & \\
\hline 13. Es fácil conseguir los materiales desde el Aula Virtual & & 0,826 & \\
\hline $\begin{array}{l}\text { 14. El proceso de uso del Aula Virtual es claro y } \\
\text { comprensible }\end{array}$ & & 0,880 & \\
\hline $\begin{array}{l}\text { 15. Utilizo con éxito el Aula Virtual para mejorar la } \\
\text { efectividad de mi trabajo como estudiante }\end{array}$ & & & 0,834 \\
\hline $\begin{array}{l}\text { 16. Utilizo con éxito el Aula Virtual para mejorar mi } \\
\text { trabajo }\end{array}$ & & & 0,798 \\
\hline $\begin{array}{l}\text { 17. Estoy satisfecho del efecto del Aula Virtual sobre mi } \\
\text { rendimiento académico }\end{array}$ & & & 0,616 \\
\hline
\end{tabular}

\section{RESULTADOS}

Los análisis de la varianza ANOVA) por género del estudiante y por razón de la titulación no encontraron diferencias significativas en ninguna de las variables. Los resultados de la regresión lineal múltiple (Tabla 2) demuestran que el desempeño percibido por los alumnos universitarios que utilizan el aula virtual SAKAI viene explicado significativamente por las tres variables planteadas. En concreto, la utilidad percibida es el factor más determinante, seguido del apoyo técnico y la facilidad de uso.

Tabla 2. Resultados de la regresión lineal múltiple

\begin{tabular}{|c|c|c|c|}
\hline $\begin{array}{c}\text { Variable dependiente }= \\
\text { desempeño percibido }\end{array}$ & $\begin{array}{c}\text { Coeficientes } \\
\text { estandarizados }\end{array}$ & $\mathrm{t}$ & Sig. \\
\cline { 2 - 3 } & Beta & \\
\hline (Constante) & & 2,153 & 0,033 \\
\hline Apoyo técnico & 0,204 & 2,652 & 0,009 \\
\hline Facilidad de uso & 0,169 & 2,338 & 0,021 \\
\hline Utilidad percibida & 0,525 & 6,885 & 0,000 \\
\hline \multicolumn{3}{|c|}{ R2 ajustado $=0,426$} \\
\hline
\end{tabular}

\section{DISCUSIÓN}

Este estudio presenta como innovación el análisis novedoso del uso del aula virtual SAKAI, que actualmente se utiliza en la Universidad de Murcia y que en su versión más reciente es similar a Moodle en sus características, para realizar un estudio inicial del efecto del apoyo técnico, utilidad percibida y facilidad de uso sobre el desempeño percibido para el caso de estudiantes universitarios españoles como medida de su satisfacción. Basándose únicamente en el modelo TAM, estudios anteriores han analizado la influencia del apoyo técnico, utilidad percibida y facilidad de uso de la herramienta Moodle sobre la actitud hacia su uso (Arteaga y Duarte, 2010) o bien sobre su satisfacción en cursos online (Kuo et al., 2014). Este trabajo estudia un modelo basado en TAM y complementado con otras teorías como la de satisfacción del usuario web que viene definida, entre otros factores, por el desempeño percibido (Mckinney et al., 2002).

Los resultados de este estudio demuestran que el rendimiento percibido por los alumnos al utilizar el campus virtual SAKAI está determinado de manera positiva y significativa por, en primer lugar, la utilidad percibida, seguido del apoyo técnico suministrado por la universidad y la facilidad de uso. El efecto de las interacciones del estudiante con los contenidos digitales del aula virtual no ha resultado significativo, ya que el constructo no presentaba niveles de fiabilidad aceptables $(\alpha$ de Cronbach $<0,8$ ) y su inclusión no mejoraba ni la varianza explicada ni el R2 ajustado del modelo.

No queremos terminar sin poner de manifiesto que este trabajo y los resultados expuestos presentan ciertas limitaciones y sesgos debido a la muestra usada y a las limitaciones del paper presentado (short 
paper), pero se trata de un estudio inicial y preliminar del modelo que se irá ampliando en posteriores análisis. El estudio además se centra en la plataforma SAKAI, por propio interés del equipo, pues actualmente es la plataforma utilizada en la Universidad de Murcia.

\section{REFERENCIAS}

Arnaldos, F., Faura, U., Lafuente, M., López, F. A., Silvia, M., Ruiz, M. (2015). Frecuencia de uso de las plataformas virtuales de enseñanza. Una comparación Moodle versus Sakai en los estudios de perfil económico. Revista de Investigación en Educación, 13 (1), 69-87.

Arteaga-Sánchez, R. \& Duarte-Hueros, A. (2010). Motivacional factors that influence the acceptance of Moodle using TAM. Computers in Human Behavior, 26(6), 1632-1640.

Attardi, S. M., \& Rogers, K. A. (2015). Design and implementation of an online systemic human anatomy course with laboratory. Anatomical Sciences Education, 8(1), 53-62.

Bainbridge, J., Melitski, J., Zahradnik, A., Lauría, E. J., Jayaprakash, S., \& Baron, J. (2015). Using learning analytics to predict at-risk students in online graduate public affairs and administration education. Journal of Public Affairs Education, 247-262.

Cheng, Y. M. (2011). Antecedents and consequences of e-learning acceptance. Information Systems Journal, 21(3), 269-299.

Chun-Kuo, Y.; Walker, A.; Schroder, K.; Belland, B. (2014). Interaction, Internet self-efficacy, and self-regulated learning as predictors of student satisfaction in online education courses. Internet and Higher Education, 20(1), 35-50.

Davis, F. D., Bagozzi, R. P., \& Warshaw, P. R. (1989). User acceptance of computer technology: a comparison of two theoretical models. Management Science, 35(8), 982-1003.

Fishbein, M. \& Azjen, I. (1975). Belief, attitude, intentions and behaviour: An introduction to theroy and research. Boston: Addison-Wesley.

Kuo, Y. C.; Walker, A. E.; Schroder, K. E. \& Belland, B. R. (2014). Interaction, Internet self-efficacy, and self-regulated learning as predictors of student satisfaction in online education courses. Internte and Higher Education, 20, 35-50.

McKinney, V., Yoon, K., \& Zahedi, F. M. (2002). The measurement of web-customer satisfaction: An expectation and disconfirmation approach. Information Systems Research, 13(3), 296-315.

McLaughlin, J. E., \& Rhoney, D. H. (2015). Comparison of an interactive e-learning preparatory tool and a conventional downloadable handout used within a flipped neurologic pharmacotherapy lecture. Currents in pharmacy teaching and learning, 7(1), 12-19.

Ngai, E. W.; Poon, J. K. \& Chan, Y. H. (2007). Empirical examination of adoption od WebCT using TAM. Computers and Education, 48 (2), 250-267.

Ong, T., Normand, M. P., \& Schenk, M. J. (2018). Using equivalence-based instruction to teach college students to identify logical fallacies. Behavioral Interventions, 33(2), 122-135.

Venkatesh, V. \& Davis, F. D. (2000). Determinants of perceived ease of use: integrating control, intrinsic motivation and emotion into technology acceptance model. Information Systems Research, 11 (4), 342-365. 